\title{
Subsurface scattering in point-based rendering
}

\section{CHOI Soo-Mi}

The Department of Computer Engineering, Sejong University in Korea and ETH Zurich in Switzerland have recently introduced a novel and simple framework for rendering subsurface scattering on surfaces represented by points. This is useful for realistically rendering a cloud of points representing translucent materials such as the human skin. This significant study is reported in Vol. 53, No. 5 of SCIENCE CHINA Information Sciences.

The point-based graphics method for rendering surfaces has gained much attention as an alternative to polygon-based graphic methods because of its simplicity and flexibility. However, current point-based techniques do not provide sufficient rendering quality for translucent materials such as the human skin. The scattering of light inside translucent materials has been extensively studied with polygon-based methods in recent years, but there has been very little focus on point-based rendering.

To accurately simulate subsurface scattering in multilayered materials, two new computation passes are added to the typical three-pass point-based rendering process. These additional passes are shadow map generation and splat-based diffusion. Splat-based diffusion is an efficient way of approximating the light diffusion phenomenon inside a material using splats. The assumption is that the diffusion of light is isotropic; therefore, its effect at a splat can be expressed as a Gaussian distribution applied to the radius of the splat. The overall diffusion effect across the surface is determined by combining the contribution of all the splats.

As the radius of a splat increases in the surface splatting process, the process slows because the number of fragments projected on the screen space has increased. It is notable that even though the number of splats with large radii decreases, the outcome does not change very much because the overlapping area between splats is large. Such observations allow us to reduce the number of splats when the amplitude of the radius is large.

Prof. Choi reported: "Although in this paper we apply the surface splatting with subsurface scattering to human facial skin, our method can be used for various translucent objects such as marble, leaves, and milk by analyzing the scattering of laser or structured light patterns. In the future, we plan to optimize the number of splats required to produce each diffuse color image and to adjust the resolution using perception-based metrics".

One reviewer commented: "This paper made a nice addition to the point-based family by adding the scattering effect". Another reviewer noted "The authors have demonstrated their method for rendering soft and semi-translucent appearances of human skin and their rendering results are quite impressive".

Prof. Choi and her student Kim are affiliated with the Laboratory of Graphics and Virtual Reality at Sejong University and Prof. Gross and Dr. Bickel are affiliated with ETH Zurich and Disney Research Zurich.

This work was supported by the National Research Foundation of Korea (Grant Nos. 2009-0073692 and F01-2009-000-10029-0) and the Swiss National Science Foundation (Grant No. 200021-130379).

See the article: Kim H J, Bickel B, Gross M, et al. Subsurface scattering using splat-based diffusion in point-based rendering. Sci China Inf Sci, 2010, 53(5): 911-919 